Original article

\title{
The challenge of deciding between home-discharge versus hospitalization in COVID-19 patients: The role of initial imaging and clinicolaboratory data
}

\author{
Abolfazl Mozafari ${ }^{\mathrm{a}}$, Mojtaba Miladinia ${ }^{\mathrm{b}}$, Ali Sabri ${ }^{\mathrm{c}}$, Fatemeh Movaseghi ${ }^{\mathrm{a}}$, \\ Mehdi Gholamzadeh Baeis ${ }^{\text {d," }}$

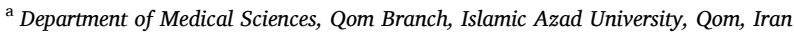 \\ ${ }^{\mathrm{b}}$ Nursing Care Research Center in Chronic Diseases, Nursing \& Midwifery School, Ahvaz Jundishapur University of Medical Sciences, Ahvaz, Iran \\ ${ }^{\mathrm{c}}$ Department of Radiology, McMaster University, Niagara Health, Ontario, Canada \\ ${ }^{\mathrm{d}}$ Department of Radiology, Imam Hossein Hospital, Shahid Beheshti University of Medical Sciences, Tehran, Iran
}

\section{A R T I C L E I N F O}

\section{Keywords:}

Human coronavirus

COVID-19

CT scan

Prognosis

Medical imaging

SARS-CoV

\begin{abstract}
A B S T R A C T
Background/Objective: It is important to predict the COVID-19 patient's prognosis, particularly in countries with lack or deficiency of medical resource for patient's triage management. Currently, WHO guideline suggests using chest imaging in addition to clinicolaboratory evaluation to decide on triage between home-discharge versus hospitalization. We designed our study to validate this recommendation to guide clinicians. This study providing some suggestions to guide clinicians for better decision making in 2020.

Methods: In this retrospective study, patients with RT-PCR confirmed COVID-19 $(\mathrm{N}=213)$ were divided in different clinical and management scenarios: home-discharge, ward hospitalization and ICU admission. We reviewed the patient's initial chest CT if available. We evaluated quantitative and qualitative characteristics of CT as well as relevant available clinicolaboratory data. Chi-square, One-Way ANOVA and Paired $t$-test were used for analysis.

Results: The finding showed that most patients with mixed patterns, pleural effusion, 5 lobes involved, total score $\geq 10$, SpO2 $\% \leq 90$, ESR $(\mathrm{mm} / \mathrm{h}) \geq 60$ and WBC $\left(10^{3} / \mu \mathrm{L}\right) \geq 8000$ were hospitalized. Most patients with Groundglass opacities only, $\leq 3$ lobes involvement, peripheral distribution, SpO2\% $\geq 95$, ESR $(\mathrm{mm} / \mathrm{h})<30$ and WBC $\left(10^{3} / \mu \mathrm{L}\right)<6000$ were home-discharged.

Conclusions: This study suggests the use of initial chest CT (qualitative and quantitative evaluation) in addition to initial clinicolaboratory data could be a useful supplementary method for clinical management and it is an excellent decision making tool (home-discharge versus ICU/Ward admission) for clinicians.
\end{abstract}

\section{Introduction}

At the beginning of December 2019, a new coronavirus (COVID-19) disease was identified in China and quickly spread to many countries around the world. Most patients develop mild symptoms such as fever, malaise, dry cough, fatigue or headache. In some patients, the disease however progresses and causes respiratory distress such as severe pneumonia, pulmonary edema, acute respiratory distress syndrome (ARDS) and multiple organ failure, and even death. ${ }^{1,2}$ In case of ARDS or organ failure, the patients may require mechanical ventilation and intensive care unit (ICU) admission. ${ }^{3}$

It is necessary to predict the patient's prognosis prior to decide on home-discharge versus hospitalization, especially in such countries as Iran (where this study took place), they have a higher rate of COVID-19 infections and the lack of sufficient resources (such as ICU beds and ventilators). Identifying the predictive indicators can also improve the outcome. ${ }^{4,5}$ Combination of imaging findings and clinicolaboratory data in the early stages of the disease are thought to be the best indicator of prognosis. Some studies suggest using chest imaging as an effective tool and appropriate prognostic factor in COVID-19 patients. ${ }^{5-8}$ Currently,

Abbreviations: COVID-19, Coronavirus disease 19; CT, Computed tomography; ICU, Intensive care unit; RT-PCR, real-time polymerase chain reaction; SpO2,

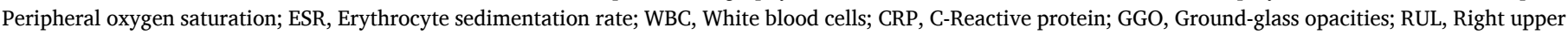
lobe; RML, Right middle lobe; RLL, Right lower lobe; LUL, Left upper lobe; LLL, Left Lower Lobe.

* Corresponding author.

E-mail address: dr.m.gholamzadeh@gmail.com (M. Gholamzadeh Baeis). 


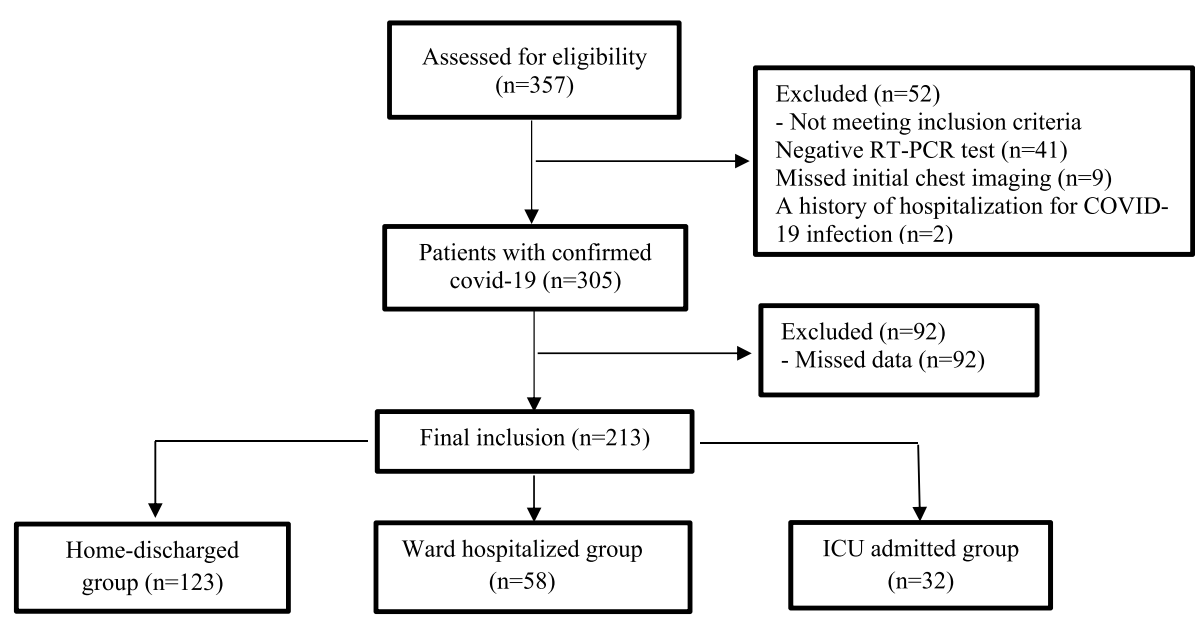

Fig. 1. Flowchart of patient selection.

world health organization (WHO) guideline also suggests using chest imaging in addition to clinicolaboratory evaluation to decide on home-discharge versus hospitalization. ${ }^{9}$ We designed our study to validate this recommendation.

This research study was possible in Iran as at early stage of the pandemic, chest imaging (computed tomography) was widely used as part of prognostic/diagnostic criteria of COVID-19 due to some limitations of diagnostic kits including a false negative result, relatively long time to determine the result, limited test kits and lack of estimation of disease severity and prognosis by real-time polymerase chain reaction (RT-PCR) test.

The study aim was to provide some suggestions for helping clinicians to decide between home-discharge versus hospitalization in COVID-19 pandemic, especially in countries with lack of sufficient resources. By identifying the important differences between various clinical groups (patients discharged home, patients admitted to the hospital wards, and patients admitted to ICU), researchers aimed to help clinicians make better treatment decisions.

\section{Material and methods}

\subsection{Design}

This study was a retrospective, single center, observational study.

\subsection{Ethics}

This retrospective study has been approved in ethics committee affiliated with Tehran Azad university of medical sciences (Ethic code: IR. IAU.PS.REC.1399.013) and did not require conscious consent from individuals and waived by the ethics committee.

\subsection{Settings and sample}

This study was conducted at the Nekoei Hedayati Forghani hospital (one of the COVID-19 patients' central hospitals) in Qom, located in central Iran. Between February 29 and April 3, 2020, 357 suspected patients admitted in the Forghani hospital and the researchers reviewed the medical records of all of them for eligibility. A total of 213 confirmed COVID-19 patients by RT-PCR entered the retrospective study and were divided into 3 groups: home-discharge (123 patients), ward hospitalized (58 patients) and ICU admitted (32 patients) [ICU admitted group: ICU beds were allocated to COVID-19 patients who were intubated and required a ventilator].

Inclusion criteria include: 1) Accessibility of an initial chest imaging, in our study it was computed tomography (CT), 2) Positive RT-PCR assay of nasal-pharyngeal swab. The patient's initial CT and initial clinicolaboratory data were reviewed ["Initial chest imaging and initial clinicolaboratory data" were meant at the time of admission to the emergency ward before triage between home-discharge versus regular ward admission versus ICU admission]. Exclusion criteria include: 1) Patients with a history of lobectomy or lung malignancy, 2) A history of hospitalization for COVID-19 infection, 3), Missing data of patients. Fig. 1 of the flowchart shows the patient selection process.

\subsection{Data collection}

The data was collected retrospectively. A checklist was designed and completed for each patient to collect data regularly. The demographic, clinical and laboratory characteristics of the patients were extracted from the medical record by two clinicians. Also, final survival status was also assessed in patients. The chest CT profile was analyzed by a radiologist and consulted by a pulmonologist who were blinded to the clinical information of the patients. Given that CT analysis may vary between radiologists (especially quantitative scoring), a second chest experienced radiologist was available for analyzes to reduce bias. We made sure that home-discharged patients were not hospitalized later. In fact, we considered the final condition of the patients for grouping.

\subsection{CT image}

All patients underwent CT-Scan by two devices [The Siemens Somatom Emotion 16-slice (Siemens Healthineers, Germany) and GE Optima540 16 Slice (GE Healthcare, USA)]. All CT-Scans are performed without contrast. The protocol used to configure the CT-Scan device was the same for all CT performed and had been regulated by a protocol notified to all imaging centers by the Iranian Society of Radiology [ISR] (COVID-19 Low-dose Thoracic CT-Scan Protocol). ${ }^{10}$ In Low-dose Thoracic CT Scan Protocol; the mean volume computed tomography dose index [CTDIvol] values, the mean dose-length product [DLP] values and Absolute cancer risk per mean cumulative effective dose values were reduced (Compared to the standard protocol), and it is also acceptable in the diagnosis of COVID-19 pneumonia). ${ }^{11}$

To perform the CT-Scan, patients are supine on the device, with their hands above their heads and holding their breath (if possible). The parameters used to set up the CT-Scan device include: Tube voltage $100-120 \mathrm{kV}$, pitch $0.8-1.5$, tube current $30-50 \mathrm{~mA}$, Thickness of Slice: 3-5 mm. All CT-Scan images were transferred to the PACS system and saved and were observed with both lung (level, $-500 \mathrm{HU}$; width, 1500 $\mathrm{HU}$ ) and mediastinal (level, $45 \mathrm{HU}$; width, $400 \mathrm{HU}$ ) window settings. 
Table 1

Baseline characteristics in different clinical groups of COVID-19 disease.

\begin{tabular}{|c|c|c|c|c|}
\hline \multirow[t]{2}{*}{ Characteristics } & \multicolumn{4}{|l|}{ Groups $(\mathrm{N}=213)$} \\
\hline & $\frac{\text { Home-discharge }}{\underline{(\mathrm{n}=123)}}$ & $\frac{\text { Hospitalized (n }}{\underline{\equiv 5 \text { ) }}}$ & $\frac{\mathrm{ICU}(\mathrm{n}=}{\underline{32)}}$ & $\underline{\mathrm{P}}$ \\
\hline \multicolumn{5}{|l|}{ Demographic } \\
\hline Age & $48.67 \pm 13.49$ & $59.27 \pm 16.16$ & $\begin{array}{l}58.03 \pm \\
15.88\end{array}$ & 0.478 \\
\hline Gender (Male) & $66(53.7 \%)$ & $24(41.4 \%)$ & $23(71.9 \%)$ & $0.021^{\mathrm{a}}$ \\
\hline \multicolumn{5}{|c|}{ Pre-existing conditions } \\
\hline Pulmonary & $13(10.56 \%)$ & $7(12.06 \%)$ & $4(12.5 \%)$ & 0.126 \\
\hline Non-pulmonary & $38(30.89 \%)$ & $16(27.58 \%)$ & $\begin{array}{l}14 \\
(43.75 \%)\end{array}$ & $0.006^{\mathrm{a}}$ \\
\hline \multicolumn{5}{|l|}{ Clinical } \\
\hline $\begin{array}{l}\text { Temperature } \\
\left(\mathrm{C}^{\circ}\right)\end{array}$ & $36.28 \pm 0.61$ & $36.82 \pm 0.55$ & $\begin{array}{l}37.02 \pm \\
0.61\end{array}$ & $<0.001^{\mathrm{a}}$ \\
\hline $\mathrm{SpO} 2$ & $95.15 \pm 3.07$ & $90.64 \pm 6.55$ & $\begin{array}{l}86.78 \pm \\
8.69\end{array}$ & $<0.001^{\mathrm{a}}$ \\
\hline Dyspnea & $76(61.8 \%)$ & $45(77.6 \%)$ & $32(100 \%)$ & $<0.001^{\mathrm{a}}$ \\
\hline \multicolumn{5}{|l|}{ Laboratory } \\
\hline ESR $(\mathrm{mm} / \mathrm{h})$ & $23.81 \pm 19.42$ & $61.83 \pm 24.16$ & $\begin{array}{l}68.50 \pm \\
25.74\end{array}$ & $<0.001^{\mathrm{a}}$ \\
\hline $\operatorname{WBC}\left(10^{3} / \mu \mathrm{L}\right)$ & $5.80 \pm 1.09$ & $8.01 \pm 4.06$ & $\begin{array}{l}10.02 \pm \\
4.04\end{array}$ & $\begin{array}{l}< \\
0.001^{\mathrm{a}}\end{array}$ \\
\hline $\operatorname{CRP}(+)$ & $2.11 \pm 1.28$ & $2.09 \pm 1.30$ & $\begin{array}{l}3.53 \pm \\
0.90\end{array}$ & 0.175 \\
\hline $\begin{array}{l}\text { Lymphocytes } \\
\text { (\%) }\end{array}$ & $28.64 \pm 12.28$ & $20.08 \pm 12.72$ & $\begin{array}{l}11.22 \pm \\
6.27\end{array}$ & $<0.001^{\mathrm{a}}$ \\
\hline \multicolumn{5}{|c|}{ Final survival status } \\
\hline Recovered & $123(100 \%)$ & $57(98.27 \%)$ & $9(28.12 \%)$ & $<0.001^{\mathrm{a}}$ \\
\hline Deceased & $0(0 \%)$ & $1(1.72 \%)$ & $\begin{array}{l}23 \\
(71.87 \%)\end{array}$ & \\
\hline
\end{tabular}

- Abbreviation: ICU (Intensive care unit admitted), SpO2 (Peripheral oxygen saturation), ESR (Erythrocyte sedimentation rate), WBC (White blood cells), CRP (C-Reactive protein).

- (Mean \pm SD or number, percent) were used.

- One-Way ANOVA and Chi-square tests were used.

${ }^{a}$ Statistically significant $(\mathrm{P}$ value $<0.05)$.

\subsection{Image interpretation}

(1) Distribution: Presence of Bilateral or Unilateral, Central or peripheral and Single or Multiple ${ }^{2}$; Type of opacities (Patterns/Density): Presence of consolidation, Crazy paving, ground-glass opacities $^{3}$; Opacities morphology: Linear or Round ${ }^{4}$; Internal structures: Air-bronchogram, cavitation, emphysema and fibrosis ${ }^{5}$; Position and number of lobes involved by difference opacities $^{6}$; Pleural thickening, Pleural effusion and Pleural retraction ${ }^{7}$; Thoracic lymphadenopathy $(\geq 10 \mathrm{~mm}$ in short-axis dimension); and ${ }^{8}$ Quantitative scoring: Total score of pulmonary involvement.

In this study, a semi-quantitative scoring system was used to estimate the extent of pulmonary involvement (Thin-Section CT Scoring). In this system, each pulmonary lobe is given a visual score of $0-5$ ( 0 without involved, 1 less than $5 \%$ involved, 2 between 5 and $25 \%$ involved, 3 between 26 and 49\% involved, 4 between 50 and 75\% involved and 5 more than $75 \%$ involved). Eventually, the score of each of the 5 lobes was added together [range 0 (no involvement) to 25 (maximum involvement)]. ${ }^{12}$

\subsection{Data analysis}

Data analysis was performed using SPSS statistical software version 16. The statistical significance level of $\mathrm{p}$-value was considered less than 0.05 . Frequency (number and percentage) was used to report categorical variables and mean and standard deviation were used to report continuous variables. Chi-square test or Fisher's exact test were used to compare categorical variables between groups. Kolmogorov-Smirnov test showed that the data had a normal distribution. One-Way ANOVA
Table 2

- CT qualitative finding in different clinical groups.

\begin{tabular}{|c|c|c|c|c|}
\hline \multirow{2}{*}{ CT features } & \multicolumn{4}{|c|}{ Groups $(\mathrm{N}=213)$} \\
\hline & $\frac{\frac{\text { Home- }}{\text { discharge }}}{\underline{(\mathrm{n}=123)}}$ & $\frac{\text { Hospitalized }}{\underline{(\mathrm{n}=58)}}$ & $\underline{\underline{\mathrm{ICU}}} \underline{\underline{\mathrm{n}=32)}}$ & $\underline{P}$ \\
\hline \multicolumn{5}{|l|}{ Distribution } \\
\hline Bilateral & $92(74.8 \%)$ & $52(89.7 \%)$ & $32(100 \%)$ & $0.001^{\mathrm{a}}$ \\
\hline $\begin{array}{l}\text { Only peripheral } \\
\text { involved }\end{array}$ & $71(57.72 \%)$ & $19(32.75 \%)$ & $2(6.25 \%)$ & $\begin{array}{c}< \\
0.001^{\mathrm{a}}\end{array}$ \\
\hline Only central involved & $0(0 \%)$ & $0(0 \%)$ & $0(0 \%)$ & - \\
\hline $\begin{array}{l}\text { Both peripheral and } \\
\text { central }\end{array}$ & $52(42.27 \%)$ & $39(67.24 \%)$ & $\begin{array}{c}30 \\
(96.75 \%)\end{array}$ & $\begin{array}{c}< \\
0.001^{\mathrm{a}}\end{array}$ \\
\hline Single lesion & $8(6.5 \%)$ & $1(1.7 \%)$ & $0(0 \%)$ & 0.143 \\
\hline Multiple lesions & $115(93.5 \%)$ & $57(98.3 \%)$ & $32(100 \%)$ & \\
\hline \multicolumn{5}{|c|}{ Type of opacities (Patterns) } \\
\hline Consolidation & $35(28.5 \%)$ & $39(67.2 \%)$ & $32(100 \%)$ & $\begin{array}{c}< \\
0.001^{\mathrm{a}}\end{array}$ \\
\hline Crazy paving & $23(18.69 \%)$ & $23(39.65 \%)$ & $\begin{array}{c}23 \\
(71.87 \%)\end{array}$ & $\begin{array}{l}< \\
0.001^{\mathrm{a}}\end{array}$ \\
\hline $\begin{array}{l}\text { Ground-glass } \\
\text { opacities } \\
\text { Opacities morpholog }\end{array}$ & $118(95.93 \%)$ & $58(100 \%)$ & $32(100 \%)$ & 0.061 \\
\hline Liner & $43(35.0 \%)$ & $41(70.7 \%)$ & $\begin{array}{c}25 \\
(78.1 \%)\end{array}$ & $\begin{array}{l}< \\
0.001^{\mathrm{a}}\end{array}$ \\
\hline Round & 116(94.3\%) & $49(84.5 \%)$ & $\begin{array}{c}29 \\
(90.6 \%)\end{array}$ & 0.057 \\
\hline \multicolumn{5}{|l|}{ Internal structures } \\
\hline Air-bronchogram & $46(37.4 \%)$ & $29(50.0 \%)$ & $\begin{array}{c}25 \\
(78.1 \%)\end{array}$ & $\underset{0.001^{\mathrm{a}}}{<}$ \\
\hline Cavitation & $0(0 \%)$ & $0(0 \%)$ & $0(0 \%)$ & - \\
\hline Emphysema & $0(0 \%)$ & $0(0 \%)$ & $0(0 \%)$ & - \\
\hline Fibrosis & $0(0 \%)$ & $0(0 \%)$ & $0(0 \%)$ & - \\
\hline \multicolumn{5}{|l|}{ Pleural involved } \\
\hline Pleural thickening & $0(0 \%)$ & $0(0 \%)$ & $0(0 \%)$ & - \\
\hline Pleural effusion & $0(0 \%)$ & $2(3.4 \%)$ & $5(15.6 \%)$ & $\underset{0.001^{\mathrm{a}}}{<}$ \\
\hline Pleural retraction & $0(0 \%)$ & $0(0 \%)$ & $0(0 \%)$ & - \\
\hline Lymphadenopathy & $0(0 \%)$ & $0(0 \%)$ & $0(0 \%)$ & - \\
\hline \multicolumn{5}{|c|}{ Number of lobes involved } \\
\hline 1 & $20(16.3 \%)$ & $5(8.6 \%)$ & $0(0 \%)$ & \\
\hline 2 & $24(19.5 \%)$ & $6(10.3 \%)$ & $0(0 \%)$ & \\
\hline 3 & $28(22.8 \%)$ & $8(13.8 \%)$ & $3(9.4 \%)$ & $\begin{array}{c}< \\
0.001^{\mathrm{a}}\end{array}$ \\
\hline 4 & $23(18.7 \%)$ & $8(13.8 \%)$ & $1(3.1 \%)$ & \\
\hline 5 & $28(22.8 \%)$ & $31(59.4 \%)$ & $\begin{array}{c}28 \\
(87.5 \%)\end{array}$ & \\
\hline \multicolumn{5}{|l|}{ Involved lobes } \\
\hline Right upper lobe & $79(64.2 \%)$ & $47(81.0 \%)$ & $\begin{array}{c}31 \\
(96.9 \%)\end{array}$ & $\begin{array}{c}< \\
0.001^{\mathrm{a}}\end{array}$ \\
\hline Right middle lobe & $1(0.8 \%)$ & $31(53.4 \%)$ & $\begin{array}{c}29 \\
(90.6 \%)\end{array}$ & $\begin{array}{c}< \\
0.001^{\mathrm{a}}\end{array}$ \\
\hline Right lower lobe & $96(78.0 \%)$ & $53(91.4 \%)$ & $\begin{array}{c}31 \\
(96.9 \%)\end{array}$ & $0.008^{a}$ \\
\hline Left upper lobe & $78(63.4 \%)$ & $47(81.0 \%)$ & $\begin{array}{c}31 \\
(96.9 \%)\end{array}$ & $\begin{array}{c}< \\
0.001^{\mathrm{a}}\end{array}$ \\
\hline Left lower lobe & $95(77.2 \%)$ & $51(87.9 \%)$ & $\begin{array}{c}31 \\
(96.9 \%)\end{array}$ & $0.016^{\mathrm{a}}$ \\
\hline
\end{tabular}

Abbreviation: CT (Computed tomography), SD (Standard deviation), n (Number), ICU (Intensive care unit admitted), GGO (Ground-glass opacities).

(Mean \pm SD or number, percent) were used.

One-Way ANOVA, Chi-square and Fisher's exact test were used.

${ }^{a}$ statistically significant $(\mathrm{P}$ value $<0.05)$.

test was used to compare continuous variables between groups. Paired $t$ test was used to compare dependent variables within each group. Independent $t$-test was also used to compare the variables between the two groups of deceased and recovered patients. Venn diagram was used to compare predominant patterns between ward hospitalized ICU admitted groups. 


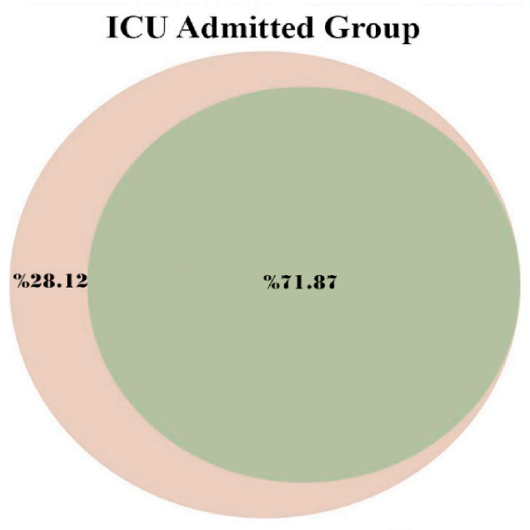

Ward Hospitalized Group

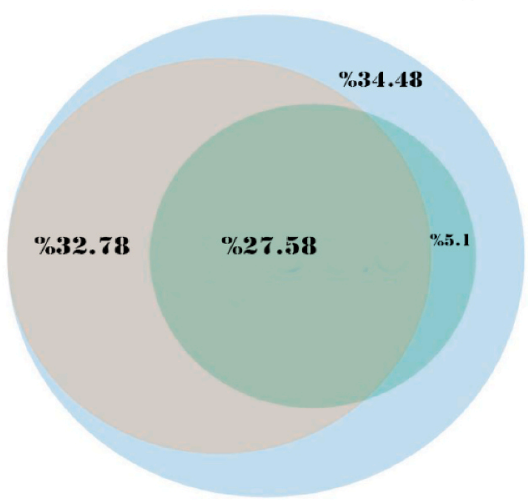

Home-Discharge Group

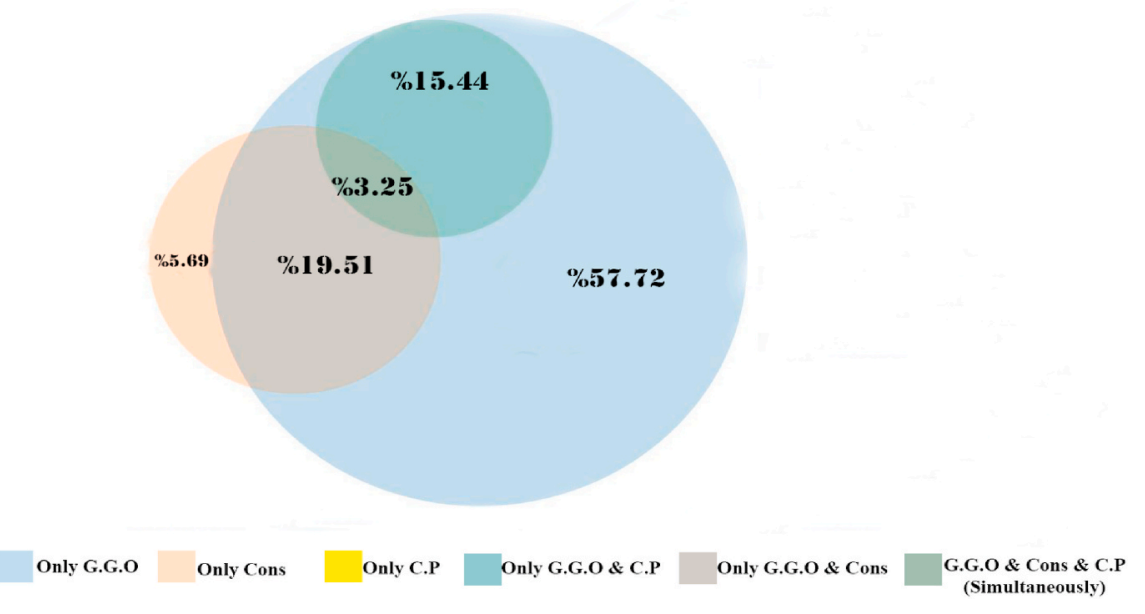

Fig. 2. Venn diagram of comparison of predominant mixed lung changes patterns between different clinical groups - Abbreviation: GGO (Ground-glass opacities), C.P (Crazy paving), Cons (Consolidation).

\section{Results}

\subsection{Baseline characteristics}

Table 1 shows baseline characteristics of patients in different clinical scenarios (Home-discharge group (57.7\%), ward hospitalized group (27.2\%) and ICU admitted group (15\%). The study included 213 patients with average age ( $48.67 \pm 13.49$, range: $18-95$ years old) and $53.1 \%(113 / 213)$ of patients were male. The majority of patients in the ICU admitted group were male and had significant difference with the other groups $(\mathrm{p}=0.021)$. Pre-existing condition (pulmonary) was also observed in all three groups, but there was no significant difference between groups. On the other hand, $43.7 \%$ of patients in the ICU admitted group had pre-existing condition (non-pulmonary) and significant difference with the other groups $(p=0.006)$.

\subsection{Clinicolaboratory data}

Clinical findings of temperature, dyspnea and $\mathrm{SpO} 2$ had a significant difference between different clinical groups (Home-discharge, ward hospitalized and ICU admitted) $(\mathrm{p}<0.001)$. Laboratory findings showed that as the severity of the disease increased, the erythrocyte sedimentation rate (ESR) and white blood cells (WBC) levels had been increased significantly and the lymphocyte counts had been decreased $(\mathrm{p}<$ 0.001). ICU admitted patients had higher C-reactive protein (CRP) levels, but this difference was not significant $(\mathrm{P}=0.175)$. The mortality rate in ICU admitted patients $(71.8 \%, 23 / 32)$ was significantly higher than in other groups $(\mathrm{p}<0.001)$. Table 1 shows Clinicolaboratory data of patients.

\subsection{Computed tomography findings (qualitative features)}

Comparison of qualitative chest CT characteristics between different clinical scenarios has been reported in 8 subgroups in Table 2. In subgroup of distribution, bilateral pulmonary involvement had a high rate in all three groups, but had been significantly increased with increasing disease severity, bilateral involvement also had been increased ( $\mathrm{p}=$ 0.001 ). In the ICU admitted group, $100 \%$ of patients had bilateral involvement. Only peripheral opacities (57\%) were observed in half of the home-discharge patients. The peripheral and central involvement was observed in the majority of patients admitted to hospital wards or ICU $(96.7 \%$ and $67.2 \%$, respectively). In none of the patients, central involvement was observed alone. Multiple lesions were also observed in all three groups and there was no significant difference between different clinical groups ( $\mathrm{p}=0.143$ ) (Table 2 ).

The most common pattern observed in different clinical groups was Ground-glass opacities (GGO). Crazy paving and consolidation patterns in the group of ward hospitalized patients were $39.65 \%$ and $67.2 \%$, respectively, however, were significantly higher in ICU admitted group than in home-discharge and ward hospitalized groups $(\mathrm{p}<0.001)$ (Table 2).

According to Venn diagram (Fig. 2), the higher the number of mixed lung changes patterns the worse the patient's prognosis. The amount of mixed patterns was observed in the ICU admitted group compared to the ward hospitalized and home-discharge group in the higher section ( $100 \%$ vs. $72.3 \%$ vs. $38.2 \%)$. In the most of home-discharge patients, ground-glass opacity alone was observed $(57.7 \%)$. In $27.5 \%$ of ward hospitalized patients, GGO alone was observed while in none of the ICU admitted patients, GGO was observed alone and all the patients in this 
Table 3

- CT quantitative finding in different clinical groups

\begin{tabular}{|c|c|c|c|c|}
\hline \multirow[t]{2}{*}{ Score } & \multicolumn{4}{|c|}{ Groups $(\mathrm{N}=213)$} \\
\hline & $\begin{array}{l}\text { Home- } \\
\text { discharge } \\
(n=123)\end{array}$ & $\begin{array}{l}\text { Hospitalized } \\
\quad(\mathrm{n}=58)\end{array}$ & ICU $(n=32)$ & $\begin{array}{c}\mathrm{P} \\
\text { (between } \\
\text { groups) }\end{array}$ \\
\hline $\begin{array}{l}\text { Total scores of } \\
\text { lower lobes } \\
\text { involved (RLL \& } \\
\text { LLL) (Range=0- } \\
\text { 10) }\end{array}$ & $3.56 \pm 2.02$ & $5.29 \pm 2.00$ & $7.68 \pm 2.26$ & $<0.001^{\mathrm{a}}$ \\
\hline $\begin{array}{l}\text { Total scores of } \\
\text { upper lobes } \\
\text { involved (RUL \& } \\
\text { LUL \& RML) } \\
\text { (Range }=0-15)\end{array}$ & $3.21 \pm 2.69$ & $5.36 \pm 3.17$ & $9.65 \pm 3.54$ & $<0.001^{\mathrm{a}}$ \\
\hline $\begin{array}{l}\text { P value } \\
\text { (Comparison of } \\
\text { lower score and } \\
\text { upper score } \\
\text { within each } \\
\text { group) }\end{array}$ & 0.089 & 0.834 & $<0.001^{\mathrm{a}}$ & \\
\hline $\begin{array}{l}\text { Total score of lung } \\
\text { involvement (5 } \\
\text { lobes) (Range }=0 \text { - } \\
25)\end{array}$ & $6.78 \pm 4.23$ & $10.66 \pm 4.96$ & $17.34 \pm 5.51$ & $<0.001^{\mathrm{a}}$ \\
\hline
\end{tabular}

Abbreviation: ICU (Intensive care unit admitted), RUL (Right upper lobe), RML (Right middle lobe), RLL (Right lower lobe), LUL (Left upper lobe), LLL (Left Lower Lobe).

(Mean \pm SD) was used.

One-Way ANOVA and paired t-test were used.

${ }^{\text {a }}$ statistically significant (P value $\left.<0.05\right)$.

group were mixed lung changes patterns. In patients who had a mix of all 3 patterns (GGO + consolidation + crazy paving), worse outcome was observed (ICU $=72 \%$ vs. ward hospitalized $=34.5 \%$ ). Homedischarge patients who had a mix of all 3 patterns were few than other groups (3.25\%).

In all three groups, there was round morphology to a greater extent, but there was no significant difference between different clinical groups ( $p=0.057)$. However, linear morphology was significantly higher with increasing disease severity ( $\mathrm{p}<0.001$ ). Fibrosis, emphysema, and cavitation were not found in any of the different clinical types. Airbronchogram was also significantly increased with increasing disease severity ( $\mathrm{p}<0.001$ ). Pleural retraction, pleural thickening, and lymphadenopathy were not observed in any group. Pleural effusion was more common in the ICU admitted group $(15.6 \%, \mathrm{p}<0.001)$, no cases were found in the home-discharge group, and only two cases $(3.4 \%)$ were observed in the ward hospitalized group (Table 2). It is noteworthy that in the studied patients, two cases of Reversed Halo Sign were observed (one case in ward hospitalized group and one case in ICU Admitted group).

The number of pulmonary lobes involved in different clinical scenarios had significant difference ( $\mathrm{p}<0.001$ ). In all three groups, 5 lobes may be involved but as the severity of the disease increased, the number of lobes involved increased. The incidence of 5 lobes involvement was higher in patients with ICU admitted and ward hospitalized groups than in home-discharge group ( $87.5 \%$ and $59.4 \%$ vs $22.8 \%$ ). Also, the involvement of one lobe and two lobes was not observed in any of the ICU admitted patients, while in the home-discharge and ward hospitalized groups, it was observed in $35.8 \%$ and $19 \%$ of patients, respectively (Table 2).

\subsection{Computed tomography findings (quantitative score)}

A comparison of quantitative score chest CT between different clinical scenarios has been reported in Table 3. The findings showed that with increasing disease severity, the mean score of pulmonary involvement had been increased significantly $(\mathrm{p}<0.001)$.
Table 4

Comparison of variables between Deceased and Recovered patients in ICU admitted group.

\begin{tabular}{|c|c|c|c|}
\hline \multirow[t]{2}{*}{$\underline{\text { Variables }}$} & \multicolumn{3}{|c|}{ Groups $(\mathrm{N}=32)$} \\
\hline & $\begin{array}{l}\text { Deceased (n } \\
=23)\end{array}$ & $\begin{array}{l}\text { Recovered (n } \\
\underline{=9)}\end{array}$ & $\underline{\mathrm{P}}$ \\
\hline \multicolumn{4}{|l|}{ Demographic } \\
\hline Age & $62.04 \pm 16.12$ & $47.77 \pm 9.92$ & $0.020^{\mathrm{a}}$ \\
\hline Gender (Male) & $16(69.6 \%)$ & $7(77.8 \%)$ & - \\
\hline \multicolumn{4}{|l|}{ Clinical } \\
\hline Temperature $\left(\mathrm{C}^{\circ}\right)$ & $37.0 \pm 0.68$ & $37.01 \pm 0.55$ & 0.687 \\
\hline $\mathrm{SpO} 2$ & $86.74 \pm 8.45$ & $86.89 \pm 9.80$ & 0.966 \\
\hline \multicolumn{4}{|l|}{ Laboratory } \\
\hline $\mathrm{ESR}(\mathrm{mm} / \mathrm{h})$ & $65.83 \pm 29.20$ & $76.50 \pm 8.54$ & 0.492 \\
\hline $\mathrm{WBC}\left(10^{3} / \mu \mathrm{L}\right)$ & $10.02 \pm 4.02$ & $10.01 \pm 1.41$ & 0.973 \\
\hline $\operatorname{CRP}(+)$ & $2.14 \pm 1.29$ & $2.00 \pm 1.41$ & 0.838 \\
\hline \multicolumn{4}{|l|}{ CT features } \\
\hline Crazy paving15(65.2\%) & $8(88.9 \%)$ & 0.383 & \\
\hline Air-bronchogram & $17(73.9 \%)$ & $8(88.9 \%)$ & 0.640 \\
\hline $\begin{array}{l}\text { Total score of lung involvement (5 } \\
\text { lobes) (Range }=0-25 \text { ) }\end{array}$ & $20.78 \pm 3.15$ & $16.00 \pm 5.69$ & $0.025^{\mathrm{a}}$ \\
\hline
\end{tabular}

- Abbreviation: ICU (Intensive care unit admitted), SpO2 (Peripheral oxygen saturation), ESR (Erythrocyte sedimentation rate), WBC (White blood cells), CRP (C-Reactive protein).

- (Mean \pm SD or number, percent) were used.

- Independent $t$-test and Fisher's exact test were used.

a Statistically significant ( $\mathrm{P}$ value $<0.05)$.

The findings also showed that the extent of the involvement in both the lower and upper lobes had been significantly increased by increasing the severity of the disease, $(\mathrm{p}<0.001)$. In the home-discharge and ward hospitalized groups, the mean score of the lower and upper lobes involvement scales were not significantly different from each other and were equally involved ( $\mathrm{P}=0.089$ and $\mathrm{P}=0.839$, respectively). However, in the ICU admitted group, the mean score of the upper lobes was significantly higher than that of the lower lobes $(\mathrm{p}<0.001)$.

Table 4 shows a comparison of different variables between deceased and recovered patients in the ICU admitted group. According to the results, most patients admitted to ICU died of the infection ( 23 form 32 , $71.9 \%$ ). Among the variables under investigation, only age and score of pulmonary involvement were significantly different between deceased and living patients. The mean age was significantly higher in deceased patients $(\mathrm{p}=0.02)$. Also, the mean score of pulmonary involvement was significantly higher in deceased patients $(\mathrm{p}=0.02)$. Crazy paving and Air-bronchogram were more common in deceased patients, but this difference was not significant. The majority of patients who died were also male $(69.6 \%)$. The other variables did not differ significantly between the two groups.

\section{Discussion}

Decision between home-discharge or hospitalization is a serious challenge for clinicians, especially in environments with a high prevalence of COVID-19 infection and limited hospital resources such as ICU beds. Therefore, it is important to determine the COVID-19 patient's prognosis for clinical management and decision making. The use of chest imaging in addition to clinicolaboratory data can play a key role to determine COVID-19 patient's prognosis, clinical management and finally better outcome for patients. This retrospective study is done to validate this statement by WHO. ${ }^{9}$

Based on our findings, qualitative and quantitative interpretation of initial chest CT in addition to initial clinicolabaratory data are useful supplementary tools to predict COVID-19 patient's prognosis. In particular, the most important parameters which suggest the patients need to be ICU/Ward admitted were mixed lung changes patterns (GGO + consolidation + crazy paving), pleural effusion, 5 lobes involvement, total score more than $10, \mathrm{SpO} 2 \% \leq 90$, ESR $(\mathrm{mm} / \mathrm{h}) \geq 60$ and WBC $\left(10^{3} / \mu \mathrm{L}\right) \geq 8000$. On the other hand, the most important parameters 


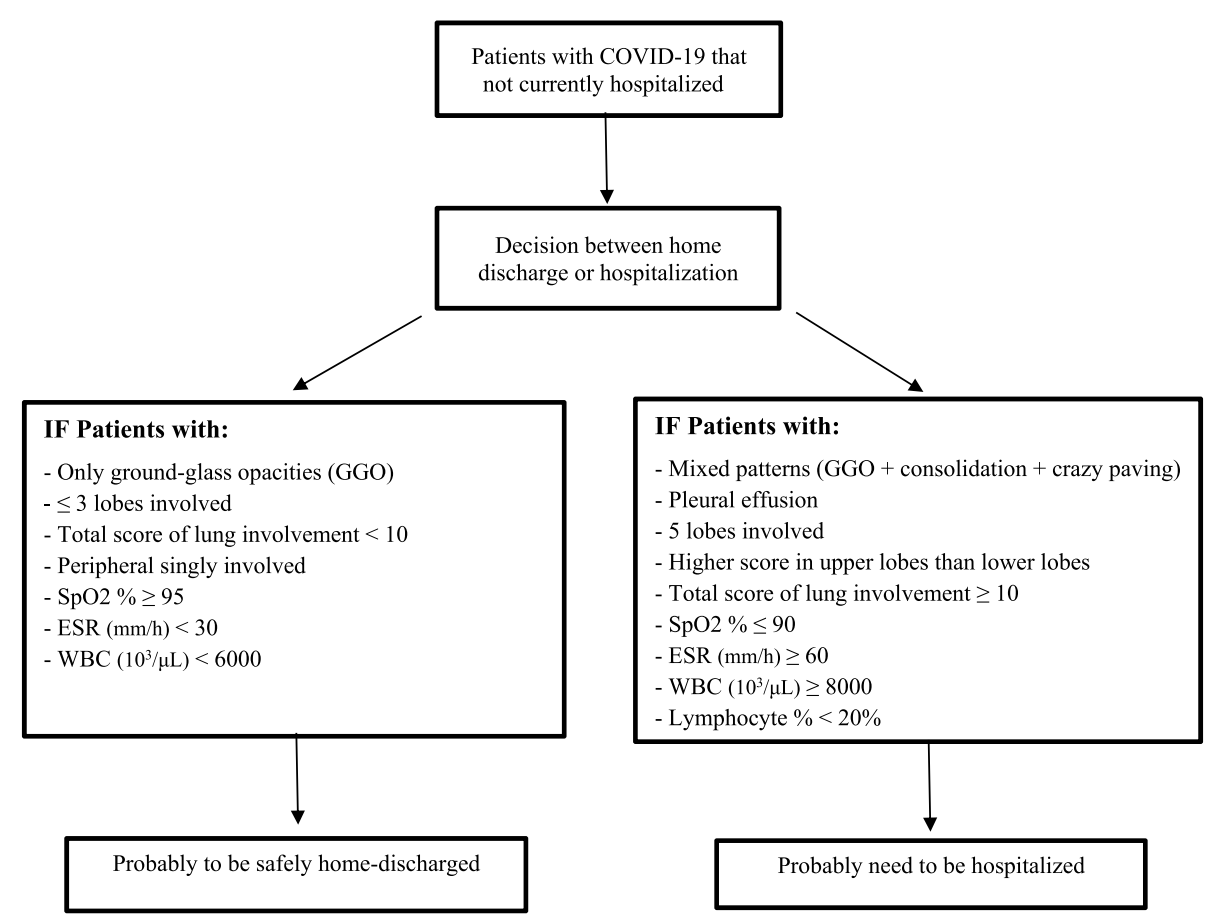

Fig. 3. Decision tree on the basis of initial CT and clinicolaboratory data in patients with COVID-19

- Abbreviation: SpO2 (Peripheral oxygen saturation), ESR (Erythrocyte sedimentation rate), WBC (White blood cells).

which suggest the patients to be safely home-discharged were groundglass opacities only, $\leq 3$ lobes involvement, only peripheral involvement, total score of less than $10, \mathrm{SpO} 2 \% \geq 95$, ESR $(\mathrm{mm} / \mathrm{h})<30$ and $\left.\mathrm{WBC} 10^{3} / \mu \mathrm{L}\right)<6000$. Fig. 3 shows decision tree on the basis of initial CT and clinicolaboratory data to guide clinicians.

Many other clinical and laboratory parameters and qualitative chest CT characteristics were not statistically significant to predict final patient's outcome (death or recovery). The findings of our study showed that the three criteria of age, sex and average quantitative score pulmonary involvement were parameters that were more reliable for predicting final patient's outcome.

Since there is no general recommendation on the treatment, imaging is still being used in many countries during the course of treatment and also as a prognostic factor. Therefore, imaging can be regarded as a very helpful alternative for management of patients with COVID-19. Although not as a first line diagnostic tool.

Identification of patients who needs to be ICU/Ward admission or home-discharge using the combination of initial CT and clinicolaboratory data can help to improvement in the planning and quality of care. Finally, Triangulation of demographic data, clinical, laboratory and radiological findings may be possible to map outcomes in more detail for a predictive tool in a larger scale investigation.

\subsection{Study limitations}

The present study also had several limitations. First, the study was single center and retrospective, and there is a possibility of bias. Second, some of the patients' clinicolaboratory and baseline information (such as weight, BMI and etc.) had been missed due to the large number of patients referred. Third, there are also limitations to the external research application, notably in countries where disease outbreak is low or the use of CT is not included in their diagnostic or management approach. Fourth, since this was a retrospective study, the authors had to use the relevant available data to register the records, thus, there was the possibility of making an error. Moreover, patient follow-up was not possible. Prospective studies and patient follow-up over time may lead to finding new solutions. Fifth, Also, the nature of retrospective study may introduce information bias or selection bias and mis-classification which we tried to avoid.

\section{Conclusion}

Decision between home-discharge versus ICU/Ward admission is a major challenge for clinicians, especially in countries with lack of resources than high volume of patients. Hence, the use of initial chest CT (qualitative characteristics and quantitative evaluation) in addition to clinicolaboratory data is a useful supplementary method to predict prognosis for clinical management and it is an excellent decision making tool (triage between home-discharge versus ICU/Ward admission) for clinicians. This confirms the current guidelines proposed by WHO.

\section{Funding}

This research did not receive any specific grant from funding agencies in the public, commercial, or not-for-profit sectors.

\section{Authors' contributions}

Study concept and design: (A.M and M. GH); Acquisition of data: (F. M and A.M); Analysis and interpretation of data: (A.M, M. GH and M.M); Statistical analysis: (M.M); Drafting of the manuscript: (M.GH, M.M and A.S); Critical revision of the manuscript for important intellectual content: (M.GH, M.M and A.S); Final approval of the version submitted for publication: (A.M, M.G and, M.M); Agreement to be accountable for all aspects of the work: (A.M, M. GH, M.M, A.S and F.M).

\section{Declarations of interest}

None.

\section{Acknowledgements}

We sincerely thank all those who have worked and are working for the management, prevention and treatment of this pandemic since the 
beginning of this pandemic, all over the world, especially doctors, nurses and other members of the medical staff.

\section{References}

1 Huang C, Wang Y, Li X, et al. Clinical features of patients infected with 2019 novel coronavirus in Wuhan, China. Lancet. 2020;395(10223):497-506.

2 Chen N, Zhou M, Dong X, et al. Epidemiological and clinical characteristics of 99 cases of 2019 novel coronavirus pneumonia in Wuhan, China: a descriptive study. Lancet (London, England). 2020;395(10223):507-513.

3 Murthy S, Gomersall CD, Fowler RA. Care for critically Ill patients with COVID-19. J Am Med Assoc. 2020;323(15):1499-1500.

4 Zhao X, Zhang B, Li P, et al. Incidence, clinical characteristics and prognostic factor of patients with COVID-19: a systematic review and meta-analysis. medRxiv. 2020, 2020.03.17.20037572.

5 Sabri A, Davarpanah AH, Mahdavi A, et al. Novel coronavirus disease 2019: predicting prognosis by using a computed tomography severity score and clinicolaboratory data. Pol Arch Intern Med. 2020;130(7-8):629-634.

6 Davarpanah AH, Asgari R, Moharamzad Y, et al. Risk factors for poor outcome in patients with severe viral pneumonia on chest CT during the COVID-19 outbreak: a perspective from Iran. SN Comprehensive Clinical Medicine. 2020. https://doi.org/ 10.1007/s42399-020-00445-3 (Epub ahead of print).

7 Cellina M, Orsi M, Toluian T, Valenti Pittino C, Oliva G. False negative chest X-Rays in patients affected by COVID-19 pneumonia and corresponding chest CT findings. Radiography. 2020;26(3):e189-e194.

8 Chen HJ, Qiu J, Wu B, et al. Early chest CT features of patients with 2019 novel coronavirus (COVID-19) pneumonia: relationship to diagnosis and prognosis. Eur Radiol. 2020;30(11):6178-6185.

9 Use of Chest Imaging in COVID-19: A Rapid Advice Guide. Geneva: World Health Organization(WHO/2019-CoV/Clinical/Radiology_imaging/2020.1): World Health Organization; 11 june 2020 [Available from: https://www.who.int/publications/i/ item/use-of-chest-imaging-in-covid-19.

10 Mahdavi A, Khalili N, Davarpanah AH, et al. Radiologic management of COVID-19: preliminary experience of the Iranian society of Radiology COVID-19 consultant group (ISRCC). Iran J Radiol. 2020;17(2), e102324.

11 Tabatabaei SMH, Talari H, Gholamrezanezhad A, et al. A low-dose chest CT protocol for the diagnosis of COVID-19 pneumonia: a prospective study. Emerg Radiol. 2020: $1-9$.

12 Pan F, Ye T, Sun P, et al. Time course of lung changes at chest CT during recovery from coronavirus disease 2019 (COVID-19). Radiology. 2020;295(3):715-721. 